\title{
Acknowledgement of priority
}

\author{
Phill Schultz
}

In a previous issue of the Gazette, [1], I showed that the number of $n \times n$ upper triangular matrices in which every row consists of a string of 0 's followed a string of 1's, and every column consists of a string of 1's followed by a string of 0's, is the Catalan number $C(n+2)$.

It has been pointed out that this result is not new. It apparently first appeared in a 1975 paper of Lou Shapiro (Howard University) in the American Mathematical Monthly, [2]. Shapiro's proof uses Dyck paths rather than bracketings, but is essentially the same as mine.

It is also curious that Shapiro used this correspondence to solve a problem similar to mine, namely classifying the ideals in the ring of $n \times n$ upper triangular matrices over a field.

\section{References}

[1] P. Schultz, Another Catalan avatar, Aust. Math. Soc. Gazette 30 (2003), 265-266.

[2] L. W. Shapiro, Upper triangular rings, ideals and Catalan numbers, Amer. Math. Monthly 82 (1975), 634-637.

School of Mathematics and Statistics, The University of Western Australia, Nedlands, WA 6009 E-mail:schultz@math.uwa.edu.au

17 December 2003. 